\title{
HOW TO BECOME NEURAL CREST: FROM SEGREGATION TO DELAMINATION
}

Aixa V. Morales ${ }^{1}$, Julio A. Barbas ${ }^{1}$ and M. Angela Nieto ${ }^{1,2}$

1Instituto Cajal, CSIC, Doctor Arce 37, 28002 Madrid and 2Instituto de Neurociencias de Alicante, CSIC-UMH, Apartado 18, Sant Joan d' Alacant, 03550 Spain

* Corresponding author: M. Angela Nieto

Tel: 34-96-5919243

Fax: 34-96-591 9561

e-mail: anieto@umh.es 
The development of the neural crest up to the stage where they leave the neural tube can be observed as a series of concatenated but independent events that involve dorsalization of the neural plate/neural tube, neural crest induction, segregation and stabilization, epithelial to mesenchymal transition and delamination. During all these processes, the nascent neural crest cells are subjected to the influence of different signals and have to overcome competition for cell fate and apoptotic signals. In addition, striking rostrocaudal differences unveil how the regulatory cascades are somehow different but still can lead to the production of bona fide neural crest cells.

The neural crest originates at the boundary between the neural plate and the prospective epidermis. Once specified, cells have to generate a population that segregated from the rest of dorsal neural cells constitute the neural crest precursor pool. Subsequently, the neural crest cells (NCCs) undergo a process of epithelium to mesenchyme transition (EMT) that will confer them the ability to migrate. The EMT involves different cellular machineries and implies deep changes in cell morphology and in the repertoire of cell surface adhesion and recognition molecules. When the EMT is complete, they delaminate from the neural folds/neural tube and migrate along characteristic pathways to differentiate into a wide variety of derivates, including neurons and glia of the peripheral nervous system, pigment cells, and craniofacial cartilage and bone (LeDouarin and Kalcheim, 1999).

Different studies have shown that diffusible signals from the ectoderm and the non-axial mesoderm, such as BMPs, BMP antagonists, Wnts, Notch, FGFs, and retinoic acid firstly confer dorsal properties to the upper part of the neural tube and direct the early steps of neural crest induction. Since recent reviews (Heeg-Truesdel and LaBonne, 2004; Huang and Saint-Jeannet, 2004; Meulemans and Bronner-Fraser, 2004) and this issue (XXX) have discussed the inductive signals extensively, we will cover the steps that run from after dorsalization of the neural epithelium to the delamination process. 


\section{A complex network of interactions occurring at early steps of neural crest development}

The signalling molecules initiate a transcriptional programmed that includes the expression of Pax3, Pax7, Msx1/2 and Zic1/3. All of them have been implicated in the development of dorsal cell types, including both NCCs and dorsal interneurons (Epstein et al., 1991; Goulding et al., 1993; Bang et al., 1997; Houzelstein et al., 1997; Mansouri and Gruss, 1998; Nakata et al., 1998). Then, specified NCCs express a differential repertoire of transcription factors that, in the context of the developing neural tube, are specifically related to the formation of the neural crest. Among them, we should mention AP-2a, Id2, Id3, FoxD3, Snail/Slug (Snail1 and Snail2, see below), Sox9, Sox10, and LSox5 (see Huang and Saint-Jeannet, 2004 and Meulemans and Bronner-Fraser, 2004, and this issue for recent comprehensive reviews). It is interesting to note that a new nomenclature has been approved by the Hugo Nomenclature Committee for Snail genes, where vertebrate Snail and Slug have been named Snail1 and Snail2, respectively (http://www.gene.ucl.ac.uk/ nomenclature/genefamily/snail.html).

Loss of AP-2 $\alpha$ results in defects in neural crest development (Schorle et al., 1996; Zhang et al., 1996) and the forced expression of AP-2a in frog embryos is sufficient to induce high level expression of genes such as Snail2 and Sox9 and the expansion of territories of neural crest differentiation (Luo et al., 2003). At differentiation stages, AP-2a participates in the generation of diverse neural crest derivatives including pigment cells, sensory neurons and cartilage (HilgerEversheim et al., 2000; Knight et al., 2003; Barrallo-Gimeno et al., 2004). However, AP-2 $\alpha$ is initially expressed throughout the ectoderm, suggesting that other factors must be involved in restricting neural crest induction to the appropriate region. Neural crest progenitors must segregate from the other dorsal neural phenotypes and recent data indicate that Id HLH transcription factors may play an important role. $I d 3$ is expressed in both the cranial and trunk neural crest progenitor pool in Xenopus embryos (Kee and Bronner-Fraser, 2005; Light et al., 2005). Kee and Bronner-Fraser propose that it is required for proliferation and survival without affecting fate. Light and co-workers report that in the absence of Id3, an excess of CNS progenitors forms at the expense of NCCs, with Id3 maintaining them in a multipotent progenitor state. Thus, although a discrepancy exists as to whether Id3 affects fate within the dorsal neural tube, it seems clear that Id3 helps to segregate and stabilize a neural crest population ready to undergo EMT. 
With respect to EMT, Snail2 was first shown to trigger EMTs in functional interference experiments. Incubation of early chick blastoderms with antisense oligonucleotides to Snail2, inhibited neural crest and mesoderm delamination from the neural tube and the early primitive streak, respectively (Nieto et al., 1994). Subsequently, defects in crest migration and lack of specific derivatives were demonstrated in the neural crest of Xenopus embryos after inhibition of Snail2 function (Carl et al., 1999; LaBonne and Bronner-Fraser, 2000). In gain-of-function experiments, Snail2 overexpression rendered the induction of $R h O B$ expression and the increase of neural crest production specifically at cranial levels (del Barrio y Nieto, 2002). A conserved role to trigger EMT during the acquisition of the invasive phenotype in tumours has been established for Snail1/Snail2 by regulating the expression of cadherins (Cano et al., 2000). Regarding FoxD3, forced expression in the neural tube of chick embryos is followed by the expansion of some neural crest within the lateral neuroepithelium, and the promotion of aberrant delamination from this region (Dottori et al., 2001). However, other authors have shown that these transformed cells do not undergo a significant EMT in similar assays (Kos et al., 2001; Cheung et al., 2005). Changes in expression patterns included the up-regulation of HNK1 and Cadherin7, and the inhibition of $\mathrm{N}$-cadherin within the neuroepithelium. However, no changes were revealed in the levels of expression of RhoB, Snail2 or Sox9. In Xenopus, both misexpression and loss of function experiments have shown that FoxD3 acts as an important positive regulator of neural crest determination, although it seems to have different requirements (Sasai et al., 2001).

Within the large family of Sox transcription factors, Sox10, Sox9 and LSox5 also participate in the delamination of NCCs and in the posterior acquisition of differential phenotypes. LSox5 expression is coincident with that of Sox10 in premigratory and most migratory NCCs, and its forced expression in the cranial neural tube of chick embryos increased the generation and delamination of NCCs (Pérez-Alcalá et al., 2004) in a similar manner as it has been shown for Snail2, AP-2a or FoxD3. Later in migratory NCCs, both Sox10 and LSox5 participate in the specific differentiation of the glial lineage (Kuhlbrodt et al., 1998; Pérez-Alcalá et al., 2004). Several lineages including melanocytes, autonomic and enteric neurons, and all subtypes of peripheral glia are missing in mice homozygous for Sox10 mutations (Britsch et al., 2001; Herbarth et al., 1998; Kapur, 1999; Southard-Smith et al., 1998). Moreover, haploinsufficiency of Sox10 results in neural crest defects that cause Waardenburg/Hirschsprung disease in humans (Pingault et al., 1998; Southard-Smith et al., 1999). Similar phenotypes and results have been reported for a fish model of this human syndrome, the mutant colourless (Dutton et al., 2001), and in both overexpression and loss of function experiments in Xenopus (Honore et al., 2003; Aoki et 
al. 2003). Sox10 is expressed in NCCs just prior to delamination and its expression is maintained in migratory cells. It is required for survival and maintenance of the multipotency of migratory NCCs before lineage segregation (Paratore et al., 2001; Kim et al., 2003).

By contrast to LSox5 and Sox10, the onset of Sox9 expression in the territory of neural crest precursors is a very early event. It closely precedes that of Snail2 in the trunk (Cheung and Briscoe, 2003). Its forced expression in the neural tube was sufficient to initiate neural crest development, but it did not efficiently induce the delamination of ectopic NCCs from the neural tube consistent with the idea that induction of neural crest segregation and initiation of an EMT are two separable events that occur in a coordinated manner, as it had been previously suggested (Newgreen and Minichielo, 1995; Sela-Donenfeld and Kalcheim, 1999). A recent study by Cheung and co-workers (Cheung et al., 2005) has added new evidences to this same concept. Gain of function experiments in the trunk neural tube with Sox9, FoxD3 and Snail2, have established differential coordinated functions for each of these factors; suggesting a key role for Sox9 in crest fate commitment and survival, and its interaction with Snail2 in triggering the EMT. FoxD3 would be involved in the regulation of cell adhesion changes, a process in which Snail2 has been commonly implied. When the forced expression of all these three factors is combined, a massive generation and delamination of NCCs is produced along the whole dorsoventral axis at expenses of other neural cell types. These results reinforce the idea that different processes during neural crest development, rather than configure a lineal cascade of hierarchical events, depict an integrated network of interactions among factors with differential coordinated functions (Fig. 1). Nevertheless, it is worth mentioning here that important differences exist in the mechanisms leading to the formation of the NCCs in head and in the trunk.

\section{Differences in neural crest development along the anteroposterior axis}

Neural crest cells originating from different levels along the anteroposterior (A-P) axis form distinct sets of derivatives (reviewed in Le Douarin and Kalcheim, 1999). Only cranial neural crest gives rise to craniofacial cartilage, while sympathetic neurons and glia are trunk-specific neural crest derivatives. It has been proposed that the origins of the rostrocaudal differences of the NCCs are established very early, at the open neural plate stage, as a result of posteriorizing signals arising from the posterior region of the embryo (Aybar and Mayor, 2002). Some of the signals involved could be Wnt and retinoic acid signals, which at least in Xenopus are responsible 
for a posteriorizing activity required for neural crest induction (Villanueva et al., 2002). However, apart from external signals, intrinsic properties determine the A-P identity of the crest cells and consequently their final destination. The A-P patterning is reflected in the expression of the Hox genes in the NCCs along the A-P axis, which confers specific cellular fates and migratory pathways upon the nascent cells (Hunt et al., 2001). In experiments of cardiac and trunk NCCs transplantation to the midbrain region, NCCs display a graded loss in developmental potential to form somatosensory neurons and cartilage along the A-P axis (Lwigale et al., 2004). Although the code of Hox expression is maintained transiently in the transplanted NCCs, it is downregulated 12 hours later. Thus, long-term differences in Hox expression cannot fully account for the rostrocaudal differences in developmental potential of the NCCs.

In addition to graded differences, accumulating evidences reveal distinct mechanisms governing the development of the neural crest in the head and the trunk. Firstly, several molecules show a different spatial and temporal pattern of expression. For instance, CD44, a receptor for extracellular matrix attachment, and the HLH inhibitor Id2 are both restricted to the neural crest of the head region (Corbel et al., 2000; Martinsen and Bronner-Fraser, 1998). Cadherin $6 b$ is quickly downregulated in the head while it is maintained in the trunk (del Barrio and Nieto, 2002). Furthermore, even at the early stages of neural crest induction, there are differences in the onset of expression of several genes. Pax3 is one of the early markers for dorsalization of the neural tube and its onset of expression is inverted in the head versus the trunk in relation to that of Snail2: Snail2 is the first to be expressed in the head, where it can induce Pax3 expression (Del Barrio and Nieto, 2002), while Pax3 expression precedes that of Snail2 in the trunk (Buxton et al., 1997). Interestingly, mice mutant for Pax3 (splotch mice) show defects in neural crest that follow an increasing rostrocaudal gradient: a reduction at the vagal and rostral trunk neural crest and the loss of neural crest emigration at the caudal thoracic, lumbar, and sacral levels (Serbedzija and McMahon, 1997).

Another proof of differences between head and trunk is reflected by the different ability of some transcription factors to generate migratory neural crest in the two regions. As such, Snail2 overexpression increases the premigratory and migratory crest population from the anterior head region up to the level of the neural tube around the fourth and fifth somites (Del Barrio and Nieto, 2002). This axial level coincides with the border between the hindbrain and the spinal cord (head and trunk), as defined by chick-quail chimeras analysis (Cambronero and Puelles, 2000). Caudal to this border, within the spinal cord, overexpression of Snail2 produces an extended area of the 
premigratory population as assessed by the ectopic expression of $R h o B$, but is not sufficient to promote a full EMT and delamination (Del Barrio and Nieto, 2002; Cheung et al., 2005). Indeed, as previously mentioned, a combination of Sox9, Snail2 and FoxD3 is needed to induce a massive production of NCCs in the trunk. However, Snail2 does not require Sox9 to execute the EMT program in the head. Interestingly, as in the case of Pax3, the onset of Sox9 expression is earlier than that of Snail2 in the trunk but after Snail2 in the head (our unpublished observations). This is in agreement with a recent analysis in mouse embryos showing that Sox9 is required for the normal generation of trunk neural crest derivatives, but its absence has little impact on the generation of cranial neural crest (Cheung et al., 2005).

Another difference between head and trunk is related to the role of BMP in delamination. BMP signalling induces neural crest delamination both in the head and the trunk but the family member involved differs. While BMP2 is responsible for neural crest delamination in the head, BMP4 has been implicated in trunk emigration (Sela-Donenfeld and Kalcheim, 1999; Kanzler et al., 2000). Although this difference can be attributed to species-specific differences, the gradient of Noggin expression along the A-P axis proposed to control the onset of neural crest delamination in the trunk (Sela-Donenfeld and Kalcheim, 1999) does not exist in the head.

Since the differences in the molecular code and mechanisms are so significant between head and trunk with respect to neural crest formation, the conclusions derived from studies carried out in only one region cannot be directly extended to the whole NCC. Unfortunately, most of the experiments involving ectopic expression or loss of function of particular genes involved in neural crest development in the chick (reviewed in Meulemans and Bronner-Fraser, 2005) are carried out either in the head or in the trunk neural crest. Furthermore, almost only the head region has been the target of study of neural crest induction and delamination in Xenopus. Thus, it would be extremely interesting to incorporate both head and trunk in the same sets of experimental designs in different vertebrates.

\section{Control of cell cycle during EMT and delamination of neural crest cells}

The Id3-expressing cells in the dorsal neural tube are specified as neural crest. They segregate from the other dorsal precursors and proliferate to generate a stabilised population ready to undergo the EMT process. EMT involves a profound reorganization of the cytoskeleton that may 
be incompatible with a high rate of cell division. Indeed, data from different systems indicate that morphogenetic movements and proliferation are not simultaneous events during rapid developmental processes such the EMTs needed for mesoderm and neural crest formation (discussed in Vega et al. 2004). With respect to the neural crest, cell proliferation is very low in Snail-expressing cells (Snail1 in the mouse and Snail2 in the chick, undergoing EMT), which in turn express very low levels of Cyclins D1 and D2. In cultured cells, Snail1 causes a blockage in the $\mathrm{G} 1$ to $\mathrm{S}$ transition by maintaining low levels of Cyclins D and high levels of p21 (Vega et al., 2004). Interestingly, NCCs synchronously enter into S phase upon delamination from the trunk neural tube (Burstyn-Cohen and Kalcheim, 2002). Both findings are compatible with Snail genes inducing EMT in the premigratory NCCs while blocking proliferation. This would allow the mesenchymal premigratory crest pool to be synchronized in G1 while undergoing changes in cell shape. Subsequently, when the EMT process is complete, cells will synchronously enter the $S$ phase during delamination. In fact, interfering with G1/S transition blocks neural crest cell delamination without affecting the expression of genes involved in early dorsal neural tube specification (Pax3, Msx1 or Cad6B) or those inducing or executing the EMT programme such as Snail2 or RhoB (Burstyn-Cohen and Kalcheim, 2002). All these data support that EMT is an independent process that can be dissociated from delamination.

The level of BMP4 signalling drives the onset of neural crest migration in the trunk neural tube through the control of G1/S transition (Burstyn-Cohen et al., 2004). Overexpressing Noggin in the trunk neural tube prevents the entry of neuroepithelial cells into the $S$ phase of the cycle at axial levels where NCCs should be migrating. Moreover, at the trunk level, BMP4 regulation of the G1/S transition is exerted via Wnt1 signalling. Inhibition of the canonical pathway of Wnt activity prevents the $\mathrm{G} 1$ to $\mathrm{S}$ transition and neural crest delamination. Additionally, Wnt1 has a separate role on the control or maintenance of the expression of dorsal neural tube specific genes as interfering with Wnt signalling pathway causes a downregulation of the expression of dorsal genes such as Pax3, Msx1 or Cad6B but not of genes expressed in specified NCCs such as RhoB, FoxD3, Sox9 or Snail2. These data, in addition to unveil additional roles for BMP and Wnt signalling, confirm that the induction of the neural crest is an event independent and downstream of the programme of dorsal specification. This explains why mice mutant for genes involved in dorsalization such as Pax3 also show defects in neural crest (Tremblay et al, 1995; Serbedzija and McMahon, 1997). 
As all the analyses described in this section have been carried out in trunk NCCs, it is not clear whether a similar mechanism controlling cell cycle operates in the cranial crest. However, the role of BMP signalling in delamination is likely to be conserved since BMP2 is expressed in the premigratory cranial NCCs in the mouse embryo, where it has an essential role in the formation and/or migration (Kanzler et al., 2000). In addition, targeted Xenopus Noggin expression in the neural crest populating the second and more caudal branchial arches results in the specific ablation of the neural crest normally originated in the targeted areas (Kanzler et al., 2000).

\section{Control of cell survival and death}

The developing hindbrain produces premigratory NCCs all along its dorsal margin, the majority of which migrate ventrally to the branchial arches. However, out of the 8 rhombomeres (r), r3 and r5 do not significantly contribute NCCS as they undergo programmed cell death in birds and mammals. Apoptosis in r3 and r5 is controlled by BMP4; acting through Msx2 (Graham et al., 1994) while a Wnt antagonist, Sfrp2, behaves as an anti-apoptotic factor (Ellies et al., 2000). The analysis of Snail1 in the mouse and Snail2 in the chicken has shown an inverse correlation between their expression and cell death in all rhombomeres and in other tissues in the embryo. Functional analysis confirms the role of Snail2 in survival, since its overexpression in the chick embryo hindbrain can rescue NCCs from apoptosis (Vega et al., 2004). The survival properties conferred by Snail2 are in agreement with the activation of survival cascades and the increase in the levels of Bcl- $X_{L}$ observed in vitro (Vega et al., 2004). The relative levels of the anti-apoptotic (Snail2 and Sfrp2) and the pro-apoptotic (Msx2) factors are important to reach a final balance of survival or death in each rhombomere. For instance, $r 2$ expresses high levels of Msx2 and Sfrp2, and low levels of Snail2. The final balance is that although many 2 NCCs die by apoptosis, many survive and generate the first stream of migration towards the first branchial arch. By contrast, $\mathrm{r} 5$ expresses high levels of Msx2 and low levels of both Snail2 and Sfrp2, resulting in a massive apoptosis.

In Xenopus embryos, an interesting analysis proposes a mechanism for the establishment of the neural crest territory involving a balance between cell survival and cell death. The same families of transcription factors (Msx and Snail) are also at play. Snail2 is expressed in the neural crest territory where it acts as an anti-apoptotic factor, and Msx1 promotes apoptosis 
at the borders of this territory (Tríbulo et al., 2004). Thus, BMP signalling and Snail proteins regulate apoptosis along the medio-lateral axis in Xenopus to refine the neural crest territory, and along the antero-posterior axis in amniotes to generate the migratory crest populations. Since the two processes occur at different stages of neural crest development, it would be interesting to know whether both are present in different vertebrates or whether there are species-specific differences.

The functional studies on the role Snail2 in neural crest survival have been carried out in the head. As already mentioned, Snail2 is also expressed in NCCs in the trunk, making it interesting to address the question as to whether it can also protect from the physiological death in the spinal cord. This is likely to be the case, since in the absence of Sox9, trunk NCCs undergo extensive apoptosis in mouse embryos (Cheung et al., 2005) leading to a massive loss of neural crest derivatives. Since the absence of Sox9 is accompanied by the downregulation of Snail1 in premigratory NCCs, these results are compatible with Snail1 also playing an important role in crest survival in the trunk. Interestingly, zebrafish embryos lacking Sox9b, the Sox9 gene expressed in the neural crest, display massive apoptosis in the central nervous system and the pharyngeal cartilage precursors (Yan et al., 2005). In addition to Sox9 and Snail2, AP-2a is also involved in conferring survival properties to the migratory neural crest population (Knight et al., 2003; Barrallo-Gimeno et al., 2004).

\section{The influence of adjacent territories on neural crest delamination}

The influence of the non-neural ectoderm, the neural plate and the paraxial mesoderm on the development of the NCCs is crucial during induction, delamination, migration and differentiation. As many of the aspects related to the influence of non-neural ectoderm and mesoderm during induction and specification are discussed in other chapters of this issue (XXX), we will focus our attention to the process that controls neural crest delamination.

The role of BMP signalling in crest delamination is controlled by the balance between the activities of BMP4 and its antagonist Noggin in the dorsal neural tube. The perturbation of the BMP4/Noggin balance by overexpression of Noggin inhibits neural crest delamination and is accompanied by a downregulation of Cadherin6B and RhoB (Sela-Donenfeld and Kalcheim, 1999). But, what does control the gradient of Noggin expression in the dorsal neural tube? It 
seems that it is a signal emanating form the dorsomedial part of the epithelial somite. The onset of neural crest migration from the neural tube is in phase with somite development (Loring and Erickson, 1987; Teillet et al., 1987). Opposite the presomitic mesoderm, NCCs are confined to the dorsal neural tube and express RhoB, Snail2, FoxD3 and Sox9 among other molecules (Liu and Jessell, 1998; Sela-Donenfeld and Kalcheim, 1999). Neural crest delamination begins facing epithelial somites and continues following somite dissociation into dermomyotome and sclerotome, when migration into the somitic mesoderm is already underway. The dorsomedial quadrant of the epithelial somite is responsible for Noggin downregulation in the dorsal neural tube, probably through the secretion of a Noggin inhibitor (Sela-Donenfeld and Kalcheim, 2000). The molecular nature of the somitic signal controlling the onset of NCCs delamination is still unknown, but the suspects are again signalling molecules already involved in other stages of neural crest development, Wnts and FGFs.

Xenopus Wnt8 from paraxial mesoderm patterns the lateral neural plate and establishes a domain, marked by Pax3 and Msx1 expression, from which neural crest will subsequently arise (Bang et al., 1999). However, the existence of such a paraxial-mesoderm derived Wnt signal in NCCs induction has been recently challenged (Monsoro-Burq et al., 2003). The authors argue that interfering with Wnt8 causes a disruption in the development of the paraxial mesoderm, with the impairment in neural crest development being just an indirect effect. They propose FGF8 as the inducer, since it can generate neural crest in the absence of mesoderm induction and without a requirement for BMP antagonists (Monsoro-Burq et al., 2003). However, the requirement of FGF8 from the paraxial mesoderm has not been directly tested yet.

After delamination has occurred, and coincidently with somite differentiation into dermomyotome and sclerotome, trunk NCCs migrate following two primary pathways: i) dorsolaterally on top of the dermomyotome (cells that will give rise to melanocytes) and ii) ventrally in a segmental fashion through the rostral half of the sclerotome of each somite (cells that will form dorsal root and sympathetic ganglia together with Schwann and adrenomedullary cells). Both pathways are controlled by paraxial mesoderm cues including cell surface-associated proteins (Eph receptors and Ephrins) and extracellular matrix components (proteoglycans and fibronectin). The second pathway is controlled by the metameric pattern of the somites (reviewed on Le Douarin and Kalcheim, 1999). 
We have just described the influence of the mesoderm on NCCs development in the trunk, but what is the situation at the head level? The head mesoderm that surrounds the cephalic neural vesicles is formed earlier than the onset of cephalic NCCs migration and thus, it cannot have the same influence as the paraxial mesoderm has in the trunk, where both tissues are formed in coordination as the body axis elongates. Besides, the differences between the trunk and the head dorsal neural tube influence the responsiveness of the head NCCs to the mesodermal influence. As already mentioned, there is not a Noggin gradient in the head neural tube controlled by somitic signals as in the trunk level and the role and position of factors such as Snail2 and Sox9 in the gene network controlling NCCs development substantially differ between head and trunk.

Nevertheless, the most obvious difference between trunk and head mesoderm is the lack of a clear and irrefutable existence of segments in the head mesoderm. Although the existence of seven mesodermal head segments (somitomeres) has been proposed in the chicken (Meier, 1981), the issue is highly controversial (Kuratani et al., 1999). More recently, it has been shown that the molecular clock that controls somitogenesis in vertebrates and cycles with the formation of each somite also operates during the formation of the anterior mesoderm. However, it only experiences two pulses of cycling gene expression. The first pulse correlates with the formation of the axial prechordal mesoderm and the second with that of all the head mesoderm (Jouve et al., 2002). If only one pulse is associated to the formation of the cranial mesoderm, the idea of it being segmented is unlikely.

NCCs emerging from the forebrain and midbrain move primarily as a broad, unsegmented sheet of cells under the ectoderm. In the hindbrain, NCCs migrate as broad streams each of which populates one branchial arch. This metameric pattern can be interpreted as reminiscent of the pattern in the trunk. However, it is not governed by the head mesoderm. Indeed, surgical manipulations of the mesoderm adjacent to hindbrain region do not influence the segmented pattern of neural crest migration (Sechrist et al., 1994). It seems that the metameric pattern of the head NCCs is controlled by intrinsic properties of the rhombomeres of the neural tube (Kontges and Lumsden, 1996) although the generation of exclusion zones adjacent to $r 3$ and r5 also contribute to it (Farlie et al., 1999). Nevertheless, it is fair saying that the rhombomeres also impose the metamery on the branchial arches muscles originated from the head mesoderm. Thus, the segmentation at the head level seems to be mainly governed by the 
neural tube that imposes it to its derived and adjacent territories, while at trunk levels, metamery is mainly imposed by the paraxial mesoderm.

Here, we have mainly reviewed the steps leading to a population of crest cells ready to delaminate and the influences from within the neural tube and from the adjacent territories to which they are subjected. All the knowledge accumulated has provided us with a plethora of transcription factors and the idea of a high complexity derived from their mutual interactions. The establishment of the precise spatio-temporal expression and activity of all these factors and the precise mapping of their interactions is a clear challenge for the near future.

\section{REFERENCES}

Aoki, Y., Saint-Germain, N., Gyda, M., Magner-Fink, E., Lee, Y., Credidio, C. and Saint-Jeannet, J. (2003). Sox10 regulates the development of neural crest-derived melanocytes in Xenopus. Dev. Biol. 259, 19-33.

Aybar, M.J. \& Mayor, R. (2002). Early induction of neural crest cells: lessons learned from frog, fish and chick. Curr Opin Genet Dev .12, 452-548.

Bang, A.G., Papalopulu, N., Kintner, C. \& Goulding, M.D. (1997). Expression of Pax-3 is initiated in the early neural plate by posteriorizing signals produced by the organizer and by posterior non-axial mesoderm. Development 124, 2075-2085.

Barrallo-Gimeno, A., Holzschuh, J., Driever, W. and Knapik, E. W. (2004). Neural crest survival and differentiation in zebrafish depends on mont blanc/tfap2a gene function. Development 131, 1463-1477.

Britsch, S. E., Goerich, D., Riethmacher, D., Peirano, R. I., Rossner, M., Nave, K. A., Birchmeier, C. \& Wegner, M. (2001). The transcription factor Sox10 is a key regulator of peripheral glial development. Genes Dev. 15, 66-78.

Burstyn-Cohen, T. \& Kalcheim, C. (2002). Association between the cell cycle and neural crest delamination through specific regulation of G1/S transition. Dev Cell. 3, 383-395.

Burstyn-Cohen, T., Stanleigh, J., Sela-Donenfeld, D. \& Kalcheim, C. (2004). Canonical Wnt activity regulates trunk neural crest delamination linking BMP/noggin signaling with G1/S transition. Development $131,5327-5339$.

Buxton, P., Hunt, P., Ferretti, P. \& Thorogood, P. (1997). A role for midline closure in the reestablishment of dorsoventral pattern following dorsal hindbrain ablation. Dev Biol. 183,150-65.

Cambronero, F. \& Puelles, L. (2000). Rostrocaudal nuclear relationships in the avian medulla oblongata: a fate map with quail chick chimeras. J Comp Neurol 427,522-45.

Cano, A., Pérez, M.A., Rodrigo, I., Locascio, A., Blanco, M.J., Del Barrio, M.G., Portillo, F. \& Nieto, M.A. (2000). The transcription factor Snail controls epithelial-mesenchymal transitions by repressing E-cadherin expression. Nat. Cell Biol. 2, 76-83. 
Carl, T.F., Dufton, C., Hanken, J. \& Klymkowsky, M.W. (1999). Inhibition of neural crest migration in Xenopus using antisense Slug RNA. Dev. Biol. 213, 101-115.

Cheung, M. \& Briscoe, J. (2003). Neural crest development is regulated by the transcription factor Sox9. Development 130, 5681-5693.

Cheung, M., Chaboissier, M.C.,Mynett, A., Hirst, E. Schedl, A. \& Briscoe, J. (2005) The transcriptional control of trunk neural crest induction, survival, and delamination. Dev. Cell 8, 179-192.

Corbel, C., Lehmann, A. \& Davison, F. (2000) Expression of CD44 during early development of the chick embryo. Mech Dev. 96, 111-114.

Del Barrio, M.G. \& Nieto, M.A. (2002). Overexpression of Snail family members highlights their ability to promote chick neural crest formation. Development 129, 1583-1593.

Dottori, M., Gross, M. K., Labosky, P. \& Goulding, M. (2001). The winged-helix transcription factor Foxd3 suppresses interneuron differentiation and promotes neural crest cell fate. Development 128, 4127-4138.

Dutton, K. A., Pauliny, A., Lopes, S.S., Elworthy, S., Carney, T.J., Rauch, J., Geisler, R., Haffter, P., Kelsh, R.N. (2001). Zebrafish colourless encodes sox10 and specifies non-ectomesenchymal neural crest fates. Development 128, 4113-4125.

Ellies, D.L., Church, V., Francis-West, P. \& Lumsden, A. (2000). The WNT antagonist cSFRP2 modulates programmed cell death in the developing hindbrain. Development 127, 5285-5295.

Epstein, D.J., Vekemans, M. \& Gruss, P. (1991). Splotch (Sp$\left.{ }^{2 H}\right)$, a mutation affecting development of the mouse neural tube, shows a deletion within the paired homedomain of Pax3. Cell 67, 767-774.

Farlie, P. G., Kerr, R., Thomas, P., Symes, T., Minichiello, J., Hearn, C. J. \& Newgreen, D. (1999). A paraxial exclusion zone creates patterned cranial neural crest cell outgrowth adjacent to rhombomeres 3 and 5. Dev. Biol. 213, 70-84.

Goulding, M.D., Lumsden, A. \& Gruss, P. (1993). Signals from the notochord regulate the region specific expression of two Pax genes in the developing spinal cord. Development 117, 1001-1017.

Graham, A., Francis-West, P., Brickell, P.\& Lumsden, A. (1994). The signalling molecule BMP4 mediates apoptosis in the rhombencephalic neural crest. Nature 372, 684-686.

Heeg-Truesdell, E. and LaBonne, C. (2004). A slug, a fox, a pair of sox: transcriptional responses to neural crest inducing signals. Birth Defects Res C Embryo Today 72, 124-139.

Herbarth, B., Pingault, V., Bondurand, N., Kuhlbrodt, K., Hermans-Borgmeyer, I., Puliti, A., Lemort, N., Goossens, M. \& Wegner, M. (1998). Mutation of the Sry-related Sox10 gene in Dominant megacolon, a mouse model for human Hirschsprung disease. Proc. Natl. Acad. Sci. U. S. A. 95, 5161-5165.

Hilger-Eversheim, K., Moser, M., Schorle, H., Buettner, R. (2000). Regulatory roles of AP-2 transcription factors in vertebrate development, apoptosis and cell-cycle control. Gene 260, 1-12.

Honore, S. M., Aybar, M. J. and Mayor, R. (2003). Sox10 is required for the early development of the prospective neural crest in Xenopus embryos. Dev. Biol 260, 79-96.

Houzelstein, D., Cohen, A., Buckingham, M. E. \& Robert, B. (1997). Insertional mutation of the mouse Msx1 homebox gene by an nlacZ reporter gene. Mech. Dev. 65, 123-133. 
Huang, X. and Saint-Jeannet, J.-P. (2004). Induction of the neural crest and the opportunities of life on the edge. Dev, Biol. 275, 1-11.

Hunt, P., Wilkinson, D. and Krumlauf. R. (1991). Patterning the vertebrate head: murine Hox 2 genes mark distinct subpopulations of premigratory and migrating cranial neural crest. Development 112, 43-50.

Kapur, R. P. (1999). Early Death of Neural Crest Cells Is Responsible for Total Enteric Aganglionosis in Sox10Dom/Sox10Dom Mouse Embryos. Ped. Dev. Pathol. 2, 559-569.

Jouve, C., limura, T. \& Pourquie O. (2002) Onset of the segmentation clock in the chick embryo: evidence for oscillations in the somite precursors in the primitive streak. Development. 129, 1107-1117.

Kanzler, B., Foreman, R.K., Labosky, P.A.\& Mallo, M. (2000). BMP signaling is essential for development of skeletogenic and neurogenic cranial neural crest. Development 127, 1095-1104.

Kee, Y. \& Bronner-Fraser, M. (2005). To proliferate or to die: role of Id3 in cell cycle progression and survival of neural crest progenitors. Genes Dev. 19, 744-755.

Kim, J., Lo, L., Dormand, E. \& Anderson, D. J. (2003). SOX10 maintains multipotency and inhibits neuronal differentiation of neural crest stem cells. Neuron $38,17-31$.

Knight, R. D., Nair, S., Nelson, S. S., Afshar, A., Javidan, Y., Geisler, R., Rauch, G.-J. and Schilling, T. F. (2003). lockjaw encodes a zebrafish tfap2a required for early neural crest development. Development 130, 5755-5768.

Kontges, G. \& Lumsden, A. (1996). Rhombencephalic neural crest segmentation is preserved throughout craniofacial ontogeny. Development. 122,3229-42.

Kos, R., Reedy, M. V., Johnson, R. L. \& Erickson, C. A. (2001). The winged-helix transcription factor FoxD3 is important for establishing the neural crest lineage and repressing melanogenesis in avian embryos. Development 128, 1467-1479.

Kuhlbrodt, K., Herbarth, B., Sock, E., Hermans-Borgmeyer, I. \& Wegner, M. (1998). Sox10, a novel transcriptional modulator in glial cells. J. Neurosci. 18, 237-250.

Kuratani, S., Horigome, N. and Hirano, S. (1999). Developmental morphology of the head mesoderm and reevaluation of segmental theories of the vertebrate head: evidence from embryos of an agnathan vertebrate, Lampetra japonica. Dev. Biol. 210, 381-400.

LaBonne, C. \& Bronner-Fraser, M. (2000). Snail-related transcriptional repressors are required in Xenopus both for induction of the neural crest and its subsequent migration. Dev. Biol. 221, 195-205.

Le Douarin, N.M. \& Kalcheim, C. (1999). The Neural Crest. Cambridge, UK: Cambridge University Press.

Light, W., Vernon, A.E., Lasorella, A., lavarone, A. \& Labonne, C. (2005). Xenopus Id3 is required downstream of Myc for the formation of multipotent neural crest progenitor cells. Development. 132, 18311841.

Liu, J.P. \& Jessell, T.M. (1998). A role for rhoB in the delamination of neural crest cells from the dorsal neural tube. Development. 125, 5055-5067.

Loring, J.F. \& Erickson, C.A. (1987). Neural crest cell migratory pathways in the trunk of the chick embryo. Dev Biol. 121, 220-236. 
Lwigale, P.Y., Conrad, G.W. \& Bronner-Fraser, M. (2004). Graded potential of neural crest to form cornea, sensory neurons and cartilage along the rostrocaudal axis. Development 131, 1979-1991.

Luo, T., Lee, Y.-H., Saint-Jeannet, J.-P. \& Sargent, T. D. (2003). Induction of neural crest in Xenopus by transcription factor AP-2A $\square$ P.roc. Natl. Acad. Sci. USA 100, 532-537.

Mansouri, A. and Gruss, P. (1998). Pax3 and Pax7 are expressed in commissural neurons and restrict ventral neuronal identity in the spinal cord. Mechanisms of Development 78, 171-178.

Martinsen, B. J. \& Bronner-Fraser, M. (1998). Neural crest specification regulated by the helix-loop-helix repressor Id2. Science 281, 988-991.

Meier, S. (1981). Development of the chick embryo mesoblast: morphogenesis of the prechordal plate and cranial segments. Dev. Biol. 83, 49-61.

Meulemans, D. \& Bronner-Fraser, M. (2004). Gene-regulatory interactions in neural crest evolution and development. Dev. Cell 7, 291-299.

Monsoro-Burq, A.H., Fletcher, R.B. \& Harland, R.M. (2003). Neural crest induction by paraxial mesoderm in Xenopus embryos requires FGF signals. Development. 130, 3111-3124.

Nakata, K., Nagai, T., Aruga, J. \& Mikoshiba, K. (1998). Xenopus Zic family and its role in neural and neural crest development. Mech. Dev. 75, 43-51.

Newgreen, D.F. \& Minichiello, J. (1995). Control of epitheliomesenchymal transformation. I. Events in the onset of neural crest cell migration are separable and inducible by protein kinase inhibitors. Dev. Biol. 170, 91-101.

Nieto, M. A., Sargent, M. G., Wilkinson, D. G. \& Cooke, J. (1994). Control of cell behaviour during development by Slug, a zinc finger gene. Science 264, 835-839.

Paratore, C., Goerich, D. E., Suter, U., Wegner, M. \& Sommer, L. (2001). Survival and glial fate acquisition of neural crest cells are regulated by an interplay between the transcription factor Sox10 and extrinsic combinatorial signaling. Development 128, 3949-3961.

Pérez-Alcalá, S. Nieto, M.A. \& Barbas, J.A. (2004). LSox5 regulates Rhob expression in the neural tube and promotes generation of the neural crest. Development 131, 4455-4465.

Pingault, V., Bondurand, N., Kuhlbrodt, K., Goerich, D. E., Prehu, M. O., Puliti, A., Herbarth, B., HermansBorgmeyer, I., Legius, E., Matthijs, G., Amiel, J., Lyonnet, S., Ceccherini, I., Romeo, G., Smith, J. C., Read, A. P., Wegner, M. \& Goossens, M. (1998) SOX10 mutations in patients with WaardenburgHirschsprung disease. Nat. Genet. 18, 171-173.

Sasai, N., Mizuseki, K. \& Sasai, Y. (2001). Requirement of FoxD3-class signaling for neural crest determination in Xenopus. Development 128, 2525-2536.

Schorle, H., Meier, P., Buchert, M., Jaenisch, R. \& Mitchell, P. J. (1996). Transcription factor AP-2 essential for cranial closure and craniofacial development. Nature 381, 235-238.

Sechrist, J., Scherson, T. \& Bronner-Fraser, M. (1994) Rhombomere rotation reveals that multiple mechanisms contribute to the segmental pattern of hindbrain neural crest migration. Development. 120, 1777-1790.

Sela-Donenfeld, D. \& Kalcheim, C. (1999). Regulation of the onset of neural crest migration by coordinated activity of BMP4 and Noggin in the dorsal neural tube. Development 126, 4749-4762. 
Sela-Donenfeld, D. \& Kalcheim, C. (2000). Inhibition of noggin expression in the dorsal neural tube by somitogenesis: a mechanism for coordinating the timing of neural crest emigration. Development 127, 4845-4854 .

Serbedzija, G. N. \& McMahon, A. P. (1997). Analysis of neural crest cell migration in Splotch mice using a neural crest-specific LacZ reporter. Developmental Biology 185, 139-147.

Southard-Smith, E.M., Kos, R., \& Pavan, W. (1998). Sox10 mutation disrupts neural crest development in Dom Hirschsprung mouse model. Nat. Genet. 18, 60-64.

Southard-Smith, E.M., Angrist, M., Ellison, J.S., Agarwala, R., Baxevanis, A.D., Chakravarti, A. \& Pavan, W.J. (1999). The Sox10(Dom) mouse: modeling the genetic variation of Waardenburg-Shah (WS4) syndrome. Genome Res. 9, 215-225.

Teillet, M.A., Kalcheim, C. \& Le Douarin, N.M. (1987). Formation of the dorsal root ganglia in the avian embryo: segmental origin and migratory behavior of neural crest progenitor cells. Dev Biol. 120, 329-347.

Tremblay, P., Kessel, M. \& Gruss, P. (1995). A transgenic neuroanatomical marker identifies cranial neural crest deficiencies associated with the Pax3 mutant Splotch. Developmental Biology 171, 317-329.

Tribulo, C., Aybar, M.J., Sanchez, S.S. \& Mayor, R. (2004). A balance between the anti-apoptotic activity of Slug and the apoptotic activity of msx1 is required for the proper development of the neural crest. Dev Biol. 275, 325-342.

Vega, S., Morales, A.V., Ocaña, O.H., Valdes, F., Fabregat, I. \& Nieto, M.A. (2004). Snail blocks the cell cycle and confers resistance to cell death. Genes Dev. 18, 1131-1143.

Villanueva, S., Glavic, A., Ruiz, P. \& Mayor, R. (2002). Posteriorization by FGF, Wnt, and retinoic acid is required for neural crest induction. Dev. Biol. 241, 289-301.

Yan, Y.L., Willoughby, J., Liu, D., Crump, J.G., Wilson, C., Miller, C.T., Singer, A., Kimmel, C., Westerfield, M. \& Postlethwait, J.H. (2005). A pair of Sox: distinct and overlapping functions of zebrafish sox9 coorthologs in craniofacial and pectoral fin development. Development 132, 1069-1083.

Zhang, J., Hagopian-Donaldson, S., Serbedzija G., Elsemore, J., Plehn- Dujowich, D., McMahon, A.P., Flavell, R.A. \& Williams, T. (1996). Neural tube, skeletal and body wall defects in mice lacking transcription factor AP-2. Nature 381, 238-241. 


\section{FIGURE LEGEND}

Figure 1. Genetic pathways involved in neural crest development. The steps represented in the figure run from the induction of dorsal properties to the neural plate/neural tube up to the completion of the epithelial to mesenchymal transition prior to delamination. After responding to the different signals, the coordinated induction of NCCs intrinsic factors leads to the acquisition of the properties of the NCCS. The arrows indicate the flow of the pathway, not direct transcriptional regulation. The different players are shown in yellow and their effects are shown in blue. 


\section{BMP Wnt Notch FGF RA}
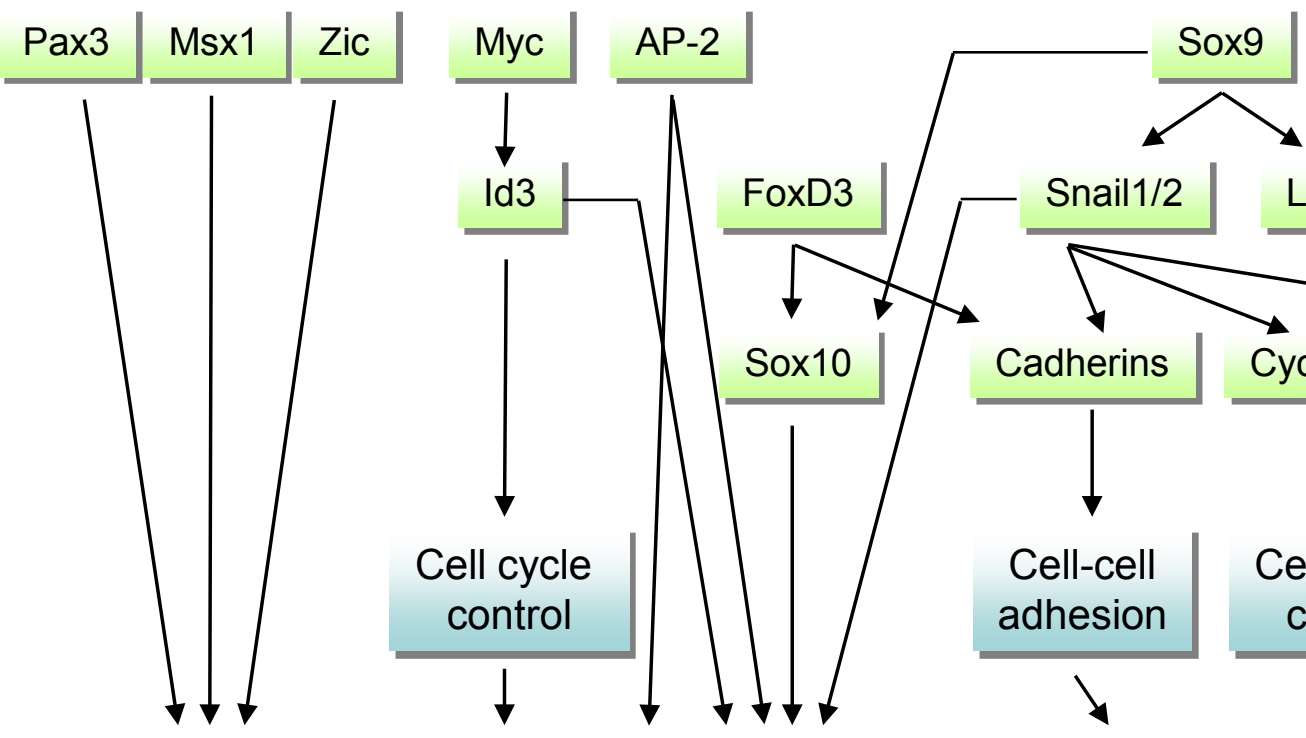

Dorsal determination
NCC segregation
NCC survival
Epithelial-mesenchymal transition

From dorsal neural specification to NCC EMT (prior to delamination)

Figure 1 